\title{
Characteristics of a primate model of focal motor cortical seizures suitable for preclinical testing of therapies like DBS
}

\author{
Shivadatta Prabhu ${ }^{1,2}$, Brigitte Piallat ${ }^{1,2}$, Annaelle Devergnas ${ }^{3}$, Thomas Blauwblomme ${ }^{4}$, \\ Ariana Sherdil $^{1,2}$, Nathalie Chivoret ${ }^{4}$, Olivier David ${ }^{1,2,5}$, Stéphan Chabardès ${ }^{1,2,6^{*}}$ \\ ${ }^{1}$ Fonctions Cérébrales et Neuromodulation, Université Joseph Fourier, Grenoble, France \\ ${ }^{2}$ Inserm, U836, Grenoble Institut des Neurosciences, Grenoble, France \\ ${ }^{3}$ Yerkes National Primate Research Center, Emory University, Atlanta, USA \\ ${ }^{4}$ Service de Neurochirurgie Pédiatrique, Hôpital Necker, Université Paris Descartes, Sorbonne Paris Cité, Paris, France \\ ${ }^{5}$ Clinique Universitaire de Neuroradiologie et IRM, Centre Hospitalier Universitaire, Grenoble, France \\ ${ }^{6}$ Clinique Universitaire de Neurochirurgie, Pôle Tête et Cou, Centre Hospitalier Universitaire, Grenoble, France \\ Email: "scharbardes@chu-grenoble.fr
}

Received 25 November 2013; revised 26 December 2013; accepted 2 January 2014

Copyright (C) 2014 Shivadatta Prabhu et al. This is an open access article distributed under the Creative Commons Attribution License, which permits unrestricted use, distribution, and reproduction in any medium, provided the original work is properly cited. In accordance of the Creative Commons Attribution License all Copyrights (C) 2014 are reserved for SCIRP and the owner of the intellectual property Shivadatta Prabhu et al. All Copyright (C) 2014 are guarded by law and by SCIRP as a guardian.

\section{ABSTRACT}

Background and objective: Generating and characterizing primate models of epileptic seizures are important for understanding pathophysiology of diseases and establishing preclinical efficacy of novel therapies like Deep Brain Stimulation. A focal motor epilepsy model is described here. Method: Seizures were obtained after intracortical penicillin injection into the motor strip through a cannula in two awake monkeys and electrocorticograms were recorded by epidural screws. Seizures were analyzed and compared for number, average duration of each seizure and total duration of ictal activity. Pharmaco-resistance for antiepileptic drug was tested by administration of $\mathrm{Di}$ azepam during seizures. Results: A motor status with seizures mimicking those seen in Kojevnikov's syndrome was easily generated several minutes after penicillin injection and lasted $24 \mathrm{~h}$ on an average. The model thus characterized appears stable and consistent. There is no significant variation between experiments in individual primate as well as between two specimens. Diazepam though reduced the total duration of seizures, failed to abolish behavioural seizures. Conclusion: This model represents a good alternative model for preclinical research aiming at testing novel therapies because seizures are obtained on demand, last up to $24 \mathrm{~h}$ after a single penicillin injection, are stable and resistant to Diazepam.

*Corresponding author.

\section{KEYWORDS}

Focal Motor Epilepsy; Primate Model; Penicillin, Preclinical Testing

\section{INTRODUCTION}

Development of novel treatment modalities for patients with intractable epilepsy especially for those who are not suitable for resective surgeries is a major research interest. Resective epilepsy surgery in eloquent cortical regions (e.g. primary motor cortex, sensori motor cortex, speech regions...) may be associated with unacceptable postoperative functional deficits. For such patients, novel therapeutic modalities such as deep brain stimulation (DBS) [1], local drug delivery in the epileptogenic zone (EZ) [2], cooling of the EZ [3] are being explored. However, pre-clinical testing of the effectiveness of these novel modalities requires well-characterized animal models mimicking various epilepsy types [4,5]. In rodents, acute and chronic models of generalized, multifocal, or limbic epilepsy have been previously described [6-8] Previous studies also reported focal neocortical seizures in rats produced by local injection of kainic acid [9,10], 4 amino pyridine [10,11], iron [12] or bicuculline $[13,14]$. However, few models are available in big mammals and in particular, models of focal neocortical epilepsy in nonhuman primate are uncommon and have not been well characterized. 


\subsection{Mechanism of Action of Penicillin}

The proconvulsant effect of penicillin (PNC) topically applied to cerebral cortex is known since 1946 [15]. PNC is a blocker of the GABAa receptors, affecting the function of cortical gamma-aminobutyric acid (GABA)-dependent interneurons [16-18], thereby leading to suppression of lateral inhibition and facilitation of the horizontal cortical activation. The neurons in the area where penicillin is injected show paroxysmal depolarizing shift [19] mimicking epileptic neurons in an epileptic focus. Previous studies suggested that intracortical injection of penicillin creates a suppression of the activity of the surrounding inhibitory interneurons that limit the spread of spikes at seizure onset $[17,18,20,21]$. Intracellular recording studies showed that penicillin induces epileptic activities in the superficial layer of the cortex and there is a vertical inhibition of the spread of spikes due to hyperpolarization of neurons in deeper layers of cortex [16].

\subsection{Penicillin among Other Epileptic Agents}

In rats, acute focal neocortical epilepsy using kayanic acid $[9,10]$, using intracortical 4-amino-pyridine (4-AP) [11] are documented. Use of local epidural bicuculline, another proconvulsant drug that also acts as a GABAblocker has been described in the past [13,14,22,23]. Focal seizures in rats can also be induced by focal electrical stimulation [24,25].

All these models have limitations that make them difficult to be used in primates. For example, the 4-AP model requires repeated injections to maintain status epilepticus. Bicuculline can lead to extremely violent seizures and high mortality. Such difficulty in administrating proconvulsant and high mortality precludes use of these agents in primates. The seizures induced by electric stimulation often get generalized, are acute and do not sustain themselves spontaneously.

Considering the mechanism of seizure induction by Penicillin and limitations of use of other proconvulsants, we choose intracortical injection of Penicillin to generate focal motor status epileticus. We also tested effect of Diazepam on the seizures in this model. Diazepam was specifically selected as antiepileptic drug in our experiments because of its allosteric modulation of GABA receptors. In this study we present electrophysiological and clinical characteristics of seizures induced by acute focal intracortical injection of PNC in the motor cortex of NHP.

\section{MATERIALS AND METHODS}

\subsection{Animals}

Study was performed on two Macaca fascicularis (CRP,
Port Louis, Mauritius), one 7 years old weighing $6.9 \mathrm{~kg}$ (Monkey A) and the other 7.5 years old weighing $7.8 \mathrm{~kg}$ (Monkey B). These 2 monkeys were included in two distinct experimental protocols that are not described in the present study, but in those protocols spontaneous seizures were recorded under similar conditions.

\subsection{Surgery}

The animals were kept fasting 10 hours before surgery. The surgery was performed under general anaesthesia with intra muscular injections of Ketamine (Imalgene, Merial laboratory, France). $0.4 \mathrm{mg}$ at induction followed by $0.2 \mathrm{mg}$ per hour) and Xylasine (Rompun, sBayer Healthcare AG, Germany) 2\%, $0.2 \mathrm{ml}$ at induction followed by $0.1 \mathrm{ml}$ per hour). Additional local scalp anaesthesia was provided by subcutaneous injection of Lignocaine with $1 \%$ Adrenalin. Prophylactic antibiotics, analgesics and anti-inflammatory drugs were delivered during perioperative period.

Surgery was performed with a stereotactic frame (David Kopf Instruments, Tujunga California USA) under intraoperative tele-radiographic control. The motor strip was first localized using bi-commissural landmarks obtained by a ventriculography performed by injecting $2 \mathrm{ml}$ water soluble iodine contrast medium (Bracco Imaging, France). Motor responses were induced by epidural cortical stimulation delivered by an external stimulator (Grass Technologies Astromed Inc. USA) and were used to confirm the location of the motor strip though a small craniotomy. Two cannula were screwed in the skull above the right motor cortex at the area representing hand to enable further injections of penicillin. Four stainless steel screws (radius of the head $2.25 \mathrm{~mm}$ length $20 \mathrm{~mm}$, Safix, Échirolles, France) were implanted bilaterally over the cranium and one additional screw was implanted close to the cannula. The screws were secured with their heads in contact with the dura. (Figure 1) Finally, a head holder (Crist Instrument Company, Hagerstown, USA) was positioned and secured with dental cement. Antero-posterior and lateral tele-Xray were used whenever necessary to check the position of the screws.

Animals were allowed to recover and monitored in the postoperative period. Food and water was provided ad libitum. Experimental induction of seizures was performed after 10 days of recovery period.

\subsection{Induction of Seizure}

Monkey was positioned on a primate chair (Crist Instruments, USA), with the head fixed to a head holder. This allowed free movements of limbs and permitted oral feeding of animals during the course of the experiments. Electrocortigcogram (ECoG) was recorded for $10 \mathrm{mi}-$ nutes prior to seizure induction. Electromyogram (EMG) 


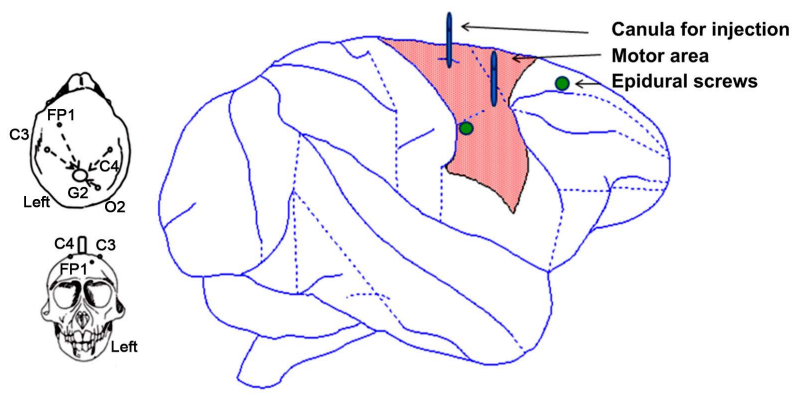

(a)

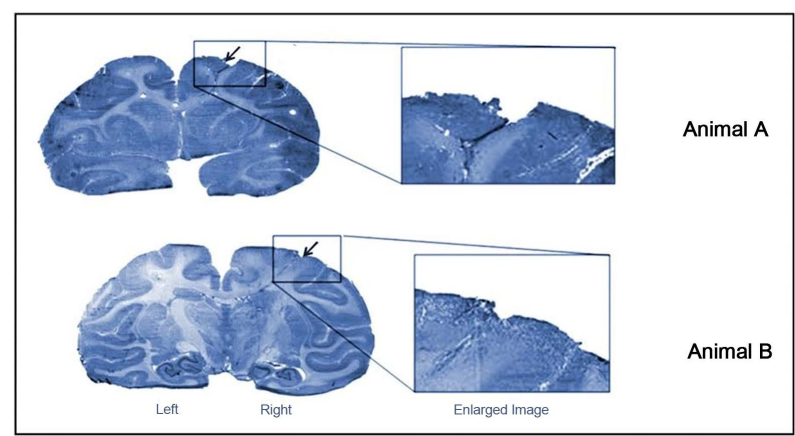

(b)

Figure 1. Schematic Representation and Histopathology. (a) Schematic representation showing location of epidural screw electrodes (black spots) and cannula-(black tubes). The canulae are located on the right motor cortex; (b) Coronal sections of brains of monkey A and monkey B showing the site of intracortical injection of Penicillin marked by a black arrow. (Crysyl Violet Stain).

of the contralateral left forelimb and hind limb was systematically monitored. PNC (PNC-G, Panpharma, Fougères, France) was diluted with sterile saline to achieve a strength of $500 \mathrm{IU} / \mathrm{l}$. Using sterile technique, a total $2500 \mathrm{IU}$ of PNC was injected at a rate of $1 \mu \mathrm{l} / \mathrm{min}$ with Hamilton syringe and pump through the cannula at a depth of $3+/-1 \mathrm{~mm}$ intracortically in the right motor cortex. After the end of injection of PNC, the injecting needle was kept in situ for 5 min before withdrawal. The dose, rate and site of intracortical injection were not modified in the series of experiments in order to allow comparison. The injections were performed twice a week. A total of 11 experiments were completed, four experiments in each monkey were completed for the purpose of comparison. In a monkey, 3 additional experiments were performed to test resistance to antiepileptic drug. In each experiment, seizures were recorded for an average of 5 hours continuously, paying attention to containment and comfort of animal in a restrained position.

\subsection{Video-ECoG-EMG Recordings}

Video-ECoG-EMG recording was performed using a commercial amplifier and software system synchronized with a video camera that can be used for standard clinical monitoring in human patients (System Plus, Micromed, Treviso Italy). ECoG and EMG signals were obtained with an analog acquisition band-pass filter between 0.05 $\mathrm{Hz}$ and $90 \mathrm{~Hz}$ and digitalized with a sampling rate of 250 Hz. A 4 channel bipolar montage between ECoG electrodes was used for analyses. ECoG/EMG synchronization with an analog video system permitted behavioral analysis of recorded seizures.

\subsection{Effect of Diazepam}

We studied the effect of Diazepam (Valium, Roche, Neuilly-Sur Seine, France) on seizures in Monkey B. A dose of $0.5 \mathrm{mg} / \mathrm{kg}$ was injected intramuscularly one hour after the intracortical injection of PNC, after the first typical seizures manifested .This dose was selected considering the recommendations made in standard primate formulary and possibility of side effects like respiratory depression in an animal restrained during experimentation. A total of 3 experiments were completed. The results were compared with the 4 experiments in the same monkey when Diazepam was not administered.

\subsection{Statistical Analysis}

The seizures were analyzed for ictal onset, evolution patterns of ECoG abnormalities and temporal resolution of the events. Two examiners analyzed the data independently and post-hoc statistical analysis found no significant inter-observer bias.

The variability in number of seizures, average duration of each seizure and total time spent during seizure in each hour were estimated for each monkey (intra subject) and between the two monkeys (inter subject). Statistical tests were performed using software GraphPad InStat3 (GraphPad Software Inc., California, USA). Inter subject variability in number of seizures, average duration of each seizure and total duration of seizure was assessed using a two tailed non parametric Wilcoxon-MannWhitney two-sample rank-sum test. The difference between datasets was quantified using the Hodges-Lehmann (HL) estimator. A nonparametric ANOVA was used for data obtained in each experiment $(n=4)$ in each experimental animal $(n=2)$. A difference was considered statistically significant for a p-value $<0.05$.

To study the effect of Diazepam, average duration of each seizure and total time spent in seizures in each hour was compared. Four control experiments and three experiments with intra muscular Diazepam were compared using a two tailed non parametric Wilcoxon-MannWhitney two-sample rank-sum test.

\subsection{Spectral Analysis}

Time-frequency analysis of 17 seizures recorded in Monkey B was performed. They were selected so that 
they contained no artifact throughout their duration, which comprised between $23 \mathrm{~s}$ and $88 \mathrm{~s}$ (average duration: $48 \mathrm{~s}$ ). ECoG signal was chosen as the monopolar derivation covering the primary motor cortex that received PNC-G (Figure 1(a)). The amplitude (square-root of power) of oscillatory activity between 1 and $100 \mathrm{~Hz}$ was obtained using standard time-frequency analysis based on the Morlet wavelet transform [26]. The first and last spikes of each discharge were used to define its onset and termination, respectively. Time window of analysis of each discharge was defined to contain at least ten seconds before seizure onset and after seizure termination. For each frequency, the amplitude was computed on 40 periods length sliding time-window, providing an effective frequency specific time resolution. Time-frequency sampling of the time frequency plane was 49 $\mathrm{ms} / 1 \mathrm{~Hz}$. Time-frequency data were normalized using the standard procedure: for each frequency, the mean of the baseline (periods of at least 10 seconds without interictal spikes chosen prior to each seizure) was subtracted from the data and then demeaned data were divided by the standard deviation of the baseline. Baseline was defined for every seizure as a period of at least 10 seconds without interictal spikes chosen just before seizure onset. Finally, the typical time-frequency pattern of recorded seizure was defined as the median value over seizures of the normalized time-frequency charts computed as such. Before median averaging, time-frequency charts were resampled linearly on a time scale to normalize seizure duration to the observed average (48 s).

\subsection{Histopathology}

Both animals were euthanized as per standard protocol after the end of experiments and brain histopathology was studied to document changes, if any, after repeated intracortical injections of penicillin (Figure 1(b)).

\section{RESULTS}

Clinical and electrical focal motor status was obtained after each single injection of PNC.

\subsection{Behavioral and Electroencephalographic Characteristics Following a Single Intracortical Penicillin Injection}

We observed three distinct electrocorticographic patterns and matching behavioral presentations.

Pattern 1: (Figure 2(a)) Interictal spikes associated with contralateral myoclonic jerks.

These interictal spikes involved single ECoG spiking with higher amplitude than baseline with brisk contraction of flexors of contralateral hind limb, sometimes associated with flexion of forelimb. The typical spike lasted 0.2 seconds, had average amplitude in the range of

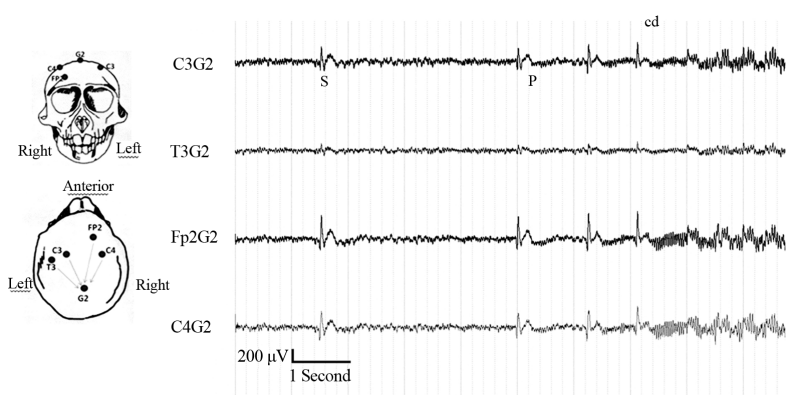

(a)

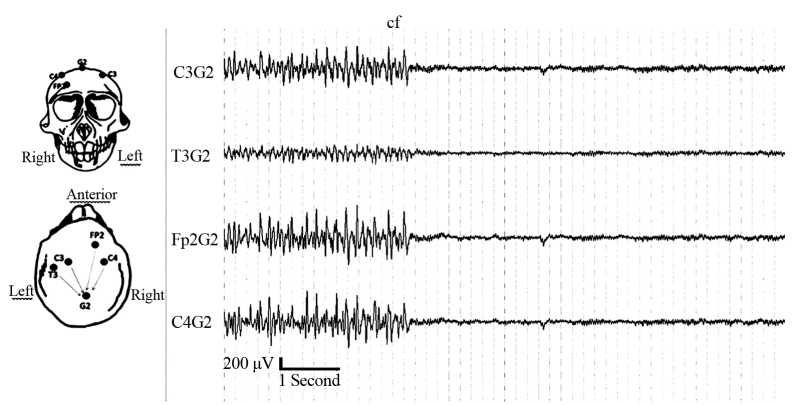

(b)

Figure 2. ECoG pattern of focal neocortical seizure after penicillin injection in the right motor cortex in primate. (a) Single interictal irregular low amplitude spikes (200 - $800 \mu \mathrm{V})$ spikes more prominent on right side associated with contralateral forelimb/hindlimb clonic movements are seen on the Left side of the ECoG.These evolve further into polyspikes at the beginning of a seizure; (b) Motor seizure consists of continuous rhythmic (Prominent on right side) high amplitude spikes (1500 to 2500 $\mu \mathrm{V}$ in amplitude, 25 to $35 \mathrm{hz}$ in frequency) Each spike is associated with contralateral hindlimb or/and forelimb clonic movement. The end of a seizure is marked by abrupt cessation of spiking. A period of about 3 to 5 seconds is noted after which the interictal spikes begin to appear again.

$200 \mu \mathrm{V}$ to $400 \mu \mathrm{V}$ with a mean frequency of $0.3 \mathrm{~Hz}$.

Pattern 2: It consisted of a train of interictal polyspikes with myoclonic contractions of contralateral hind limb occurring at an average of 3 to 6 in succession. Polyspikes lasted for less than 3 seconds, had average amplitude in the range of $200-400 \mu \mathrm{V}$.

Pattern 3: Motor seizure (Figure 2(b))

This pattern followed preictal myoclonus that evolved into a sustained seizure lasting between 5 seconds and 120 seconds (mean $49+/-8$ s.). The beginning of seizure was marked by ECoG spikes, similar to interictal ones , with amplitude in the range of $200 \mu \mathrm{V}$ to $800 \mu \mathrm{V}$, at a frequency of 0.5 to $1 \mathrm{~Hz}$ and lasting less than $5 \mathrm{~s}$. This was followed by ictal discharge on ECoG with a frequency in the range of 20 to $25 \mathrm{~Hz}$. The amplitude increased to a range $1500 \mu \mathrm{V}$ to $2500 \mu \mathrm{V}$ (mean $1850+/-$ $372.5 \mu \mathrm{V}$ ) during the seizure. The end of seizure was marked by abrupt return of ECoG to baseline.

Time-frequency chart of the normalized amplitude of primary motor cortex activity during motor seizures 
(Figure 3) confirmed the primary role of beta oscillations $(12-25 \mathrm{~Hz})$ that started $3 \mathrm{~s}$ after seizure onset and remained steady until seizure termination. Seizure onset was characterized by a brief vertical column of activity between 1 and $70 \mathrm{~Hz}$ that corresponds to first large spike. During the three seconds following the first spike, a fast drift of low-gamma activity, from $40 \mathrm{~Hz}$ to $25 \mathrm{~Hz}$, could be observed. Low-gamma activity became broadly distributed in the second part of the seizure, and kept increasing until seizure termination, particularly between $25-40 \mathrm{~Hz}$.

This was accompanied by appearance of $4 \mathrm{~Hz}$ activity. These two features actually translate in the time frequency domain the bursty ECoG patterns (4 Hz be-tagamma bursts) that could be observed at the end of seizures.

The clinical picture of a typical seizure demonstrated initial tonic contraction of contralateral hind limb often associated with involvement of contralateral fore limb. This was followed by clonic movements of contralateral limbs. It ended by abrupt cessation of motor activity with concurrent return to baseline of ECoG signal.

\subsection{Temporal Evolution of Interictal Spikes and Seizures}

In all experiments, a consistent succession of ECoG patterns in relation to seizure activity could be observed.

\subsubsection{Occurrence of Interictal Spikes}

Typically, interictal spikes were apparent within the 2 to 5 min following intracortical injection of penicillin. Interictal spikes and myoclonic jerks were most numerous during the first hour (Figure 4) and were partially replaced by seizures afterwards. The number of interictal spikes gradually decreased in following hours, but they

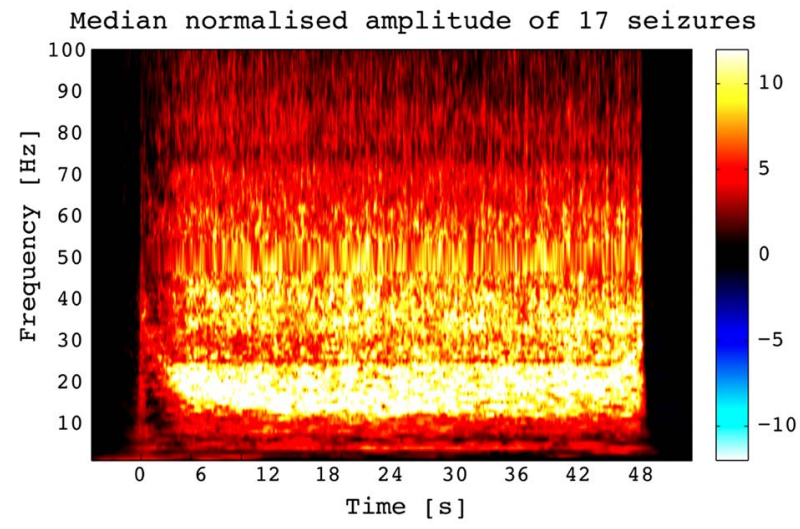

Figure 3. Time-frequency chart of the normalized amplitude of primary motor cortex activity during motor seizures. Color codes for the median value of 17 seizures, expressed in standard deviation of baseline (z-score). Time frequency analysis of seizures shows predominant Beta band activity.

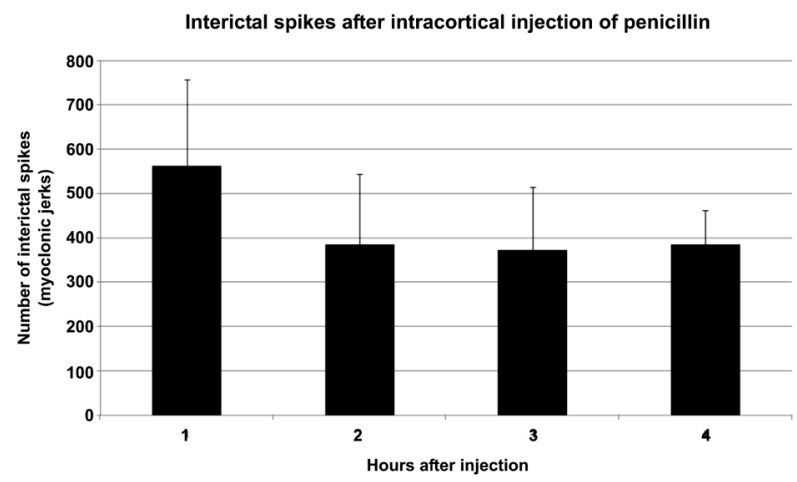

Figure 4. Interictal spikes. Average number of interictal spikes in one specimen during experiments. The number was maximum in the first hour after intra cortical injection of Penicillin. Thereafter it reached a plateau that lasted for several hours. $\mathrm{X}$-axis: Hours after intracortical injection; $\mathrm{Y}$-axis: Number of interitctal spikes.

could still be clinically detected at the 8th hour post injection.

\subsubsection{Evolution of Seizures}

The first typical seizure appeared from $30 \mathrm{~min}$ to $54 \mathrm{~min}$ after the injection. The seizure duration and severity (in terms of involvement of muscle groups of the limbs in observed tonic clonic activity) increased gradually during subsequent hours, reaching a peak during the third hour before tapering off in the fifth hour of the post injection period. Even after removal of animal from the restrain chair, the behavioral seizures could be observed till seventh hour. In every experiment, no behavioral or ECOG abnormality could be detected the next day.

\subsection{Seizure Characterization}

1) Monkey A

The total time spent in seizures and average duration of seizure in each hour in four experiments is graphically described below (Figure 5).

The average number of seizures was $8.5( \pm 4)$ in the first hour and gradually increased in the second and third hour before decreasing to $10.5( \pm 9.8)$ in the fourth hour. The average time spent in seizures was around $20 \%$ of total duration for the first hour, around 30\% in second and third hour and $40 \%$ of total duration in the fourth hour. The average duration of each seizure was 61.55 $( \pm 38.84)$ in the first hour and was $73.07 \mathrm{~s}( \pm 23.4 \mathrm{~s})$ in last hour.

2) Monkey B

The total time spent in seizures and average duration of seizure in each hour in four experiments is graphically described below (Figure 6).

The average number of seizures was $11(+/-1.7)$ in first hour, increased in second and third hours before decreasing to $21.5(+/-8.6)$ in the 4 th hour. The average 
Total time spent in seizure (Animal A)

(A)

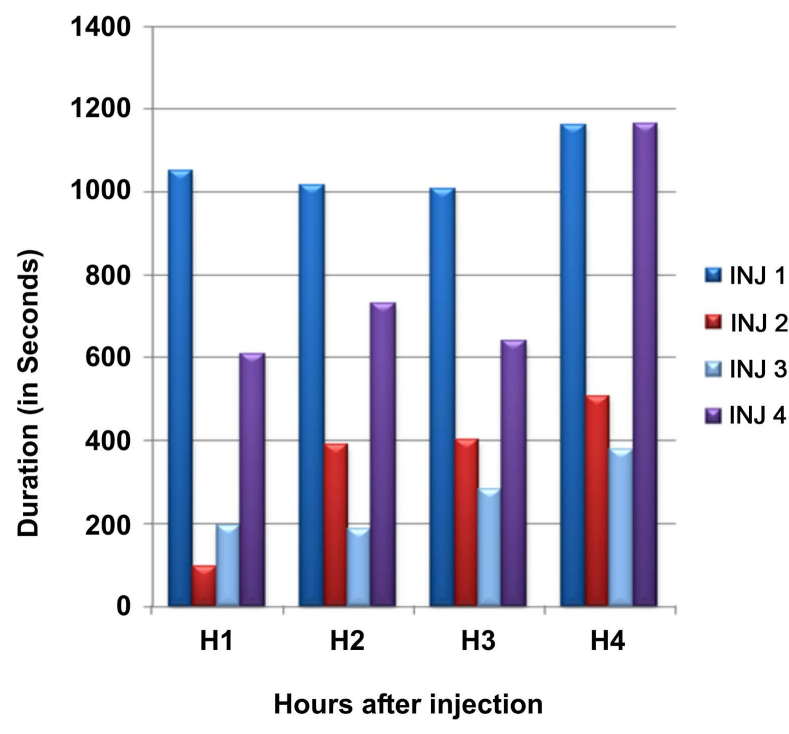

Average duration of seizure (Animal A)

(B)

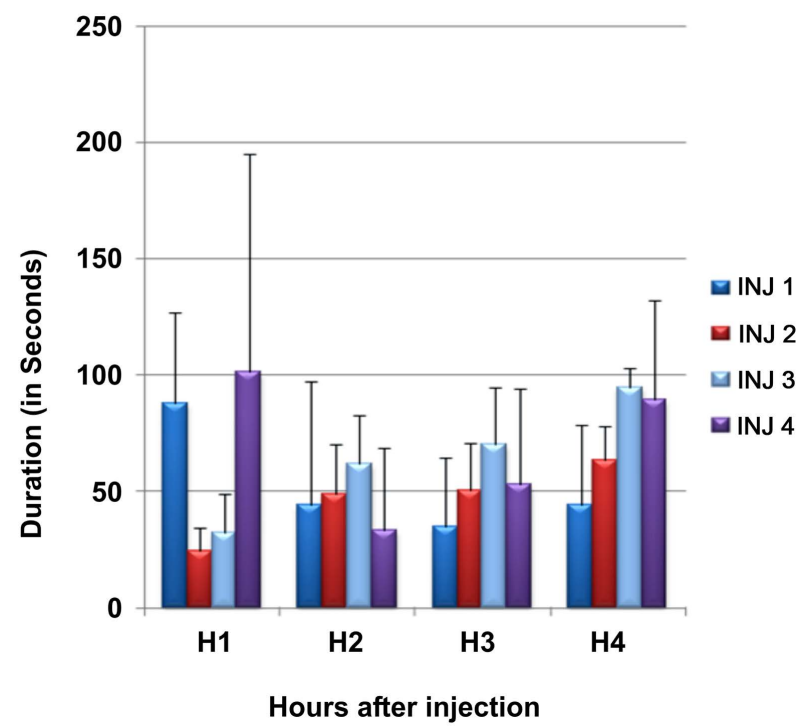

Figure 5. Seizures in monkey A. Part A of figure shows total time spent in seizures and part B shows average duration of seizure during each hour in each experiment (total 4) (Inj: injection of intracortical Penicillin). X-axis: Hours after intracortical injection; Y-axis: Total time in spent in seizures in seconds.

time spent in seizures was around $15 \%$ of total duration for the first hour, around $28.30 \%$ and $29.70 \%$ in the second and third hour respectively and around $27 \%$ of total duration)in the fourth hour. The average duration of each seizure was $31.48(+/-10.1)$ in the first hour and $48.35 \mathrm{~s}(+/-15)$ in the fourth hour.

\section{Total time spent in seizure (Animal B)}

(A)

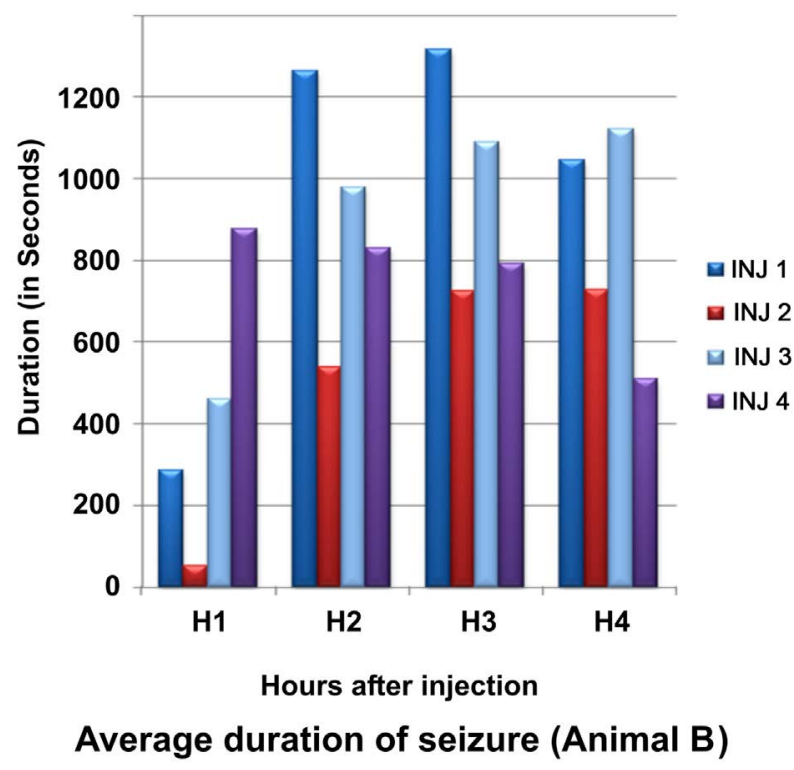

(B)

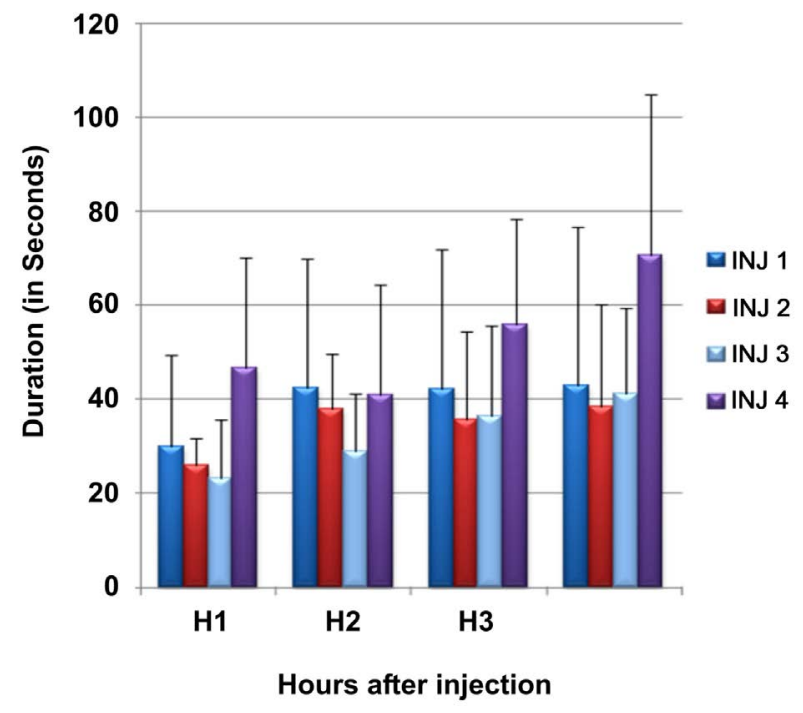

Figure 6. Seizures in monkey B. Part A of graph shows total time spent in seizures and part B shows average duration of seizure seizure during each hour in each experiment (total 4) (Inj: injection of intracortical Penicillin). $\mathrm{X}$ axis: Hours after intracortical injection; $\mathrm{Y}$ axis: Total time in spent in seizures in seconds.

\subsection{Intersubject Variability}

The pattern of seizure evolution was same in both specimens. The comparison between the two monkeys showed that average duration of seizures $(p \geq 0.05)$ (Figure 7(A)), number of seizures $(p \geq 0.05)$ (Figure $7(B))$, and total time spent during seizure $(p \geq 0.05)$ did not differ significantly. 


\section{Average duration of each seizure Animal A and B}

(A)

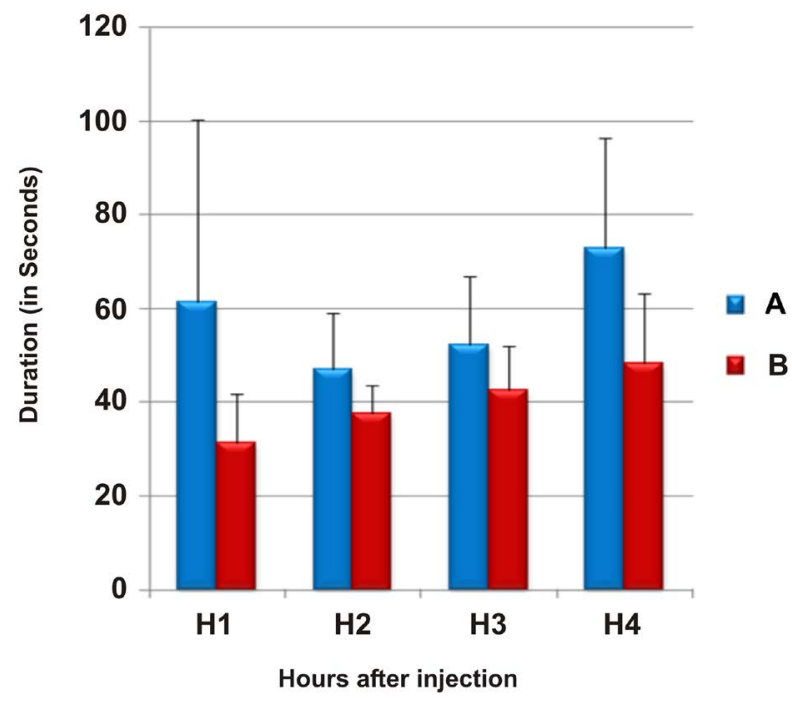

Number of seizures in each hour Animal A and B

(B)

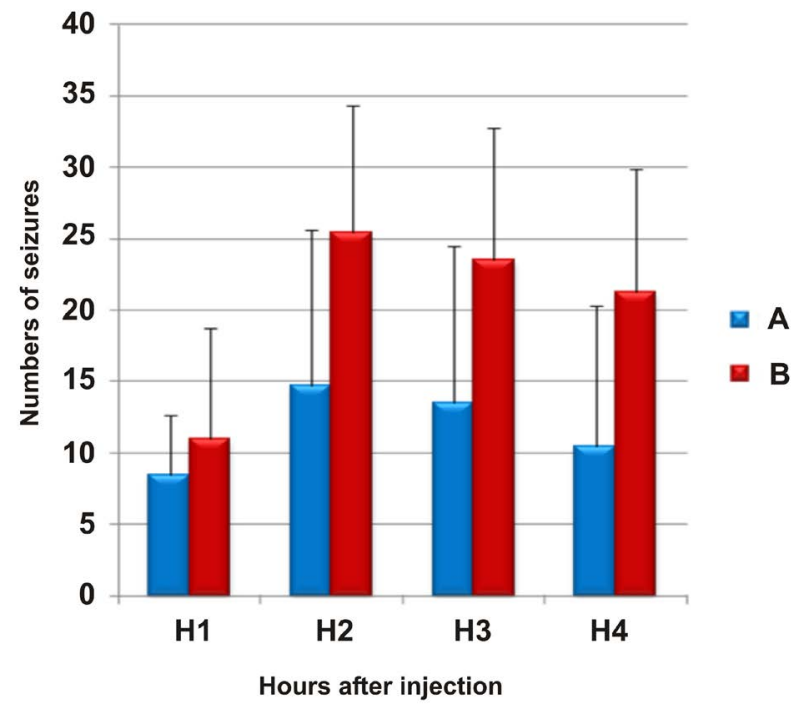

Figure 7. Comparison between the two monkeys, A and B. Part A of figure shows comparison of average duration of seizure between two monkeys. Part B of figure shows comparison of number of seizures between two monkeys. $\mathrm{X}$ axis: Hours after intracortical injection; $\mathrm{Y}$ axis: Total time in spent in seizures in seconds; Monkey A (grey bars) Monkey B (black bars).

\subsection{Effect of Diazepam on Seizures}

In Monkey B, compared to control experiments, administration of Diazepam decreased the total time spent in seizures (Figure 8(a)). However it remained steady (at around 200 seconds in each hour) throughout. The difference between average duration of seizure in each hour between control and experiments that included diazepam administration, was not statistically significant $(\mathrm{p} \geq 0.05)$

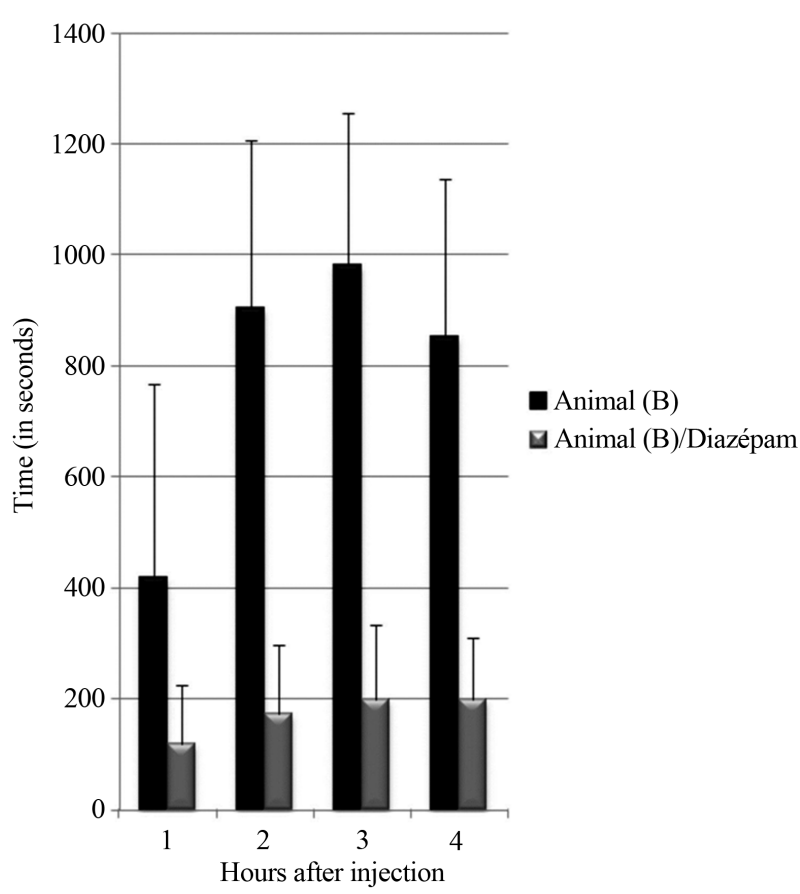

(a)

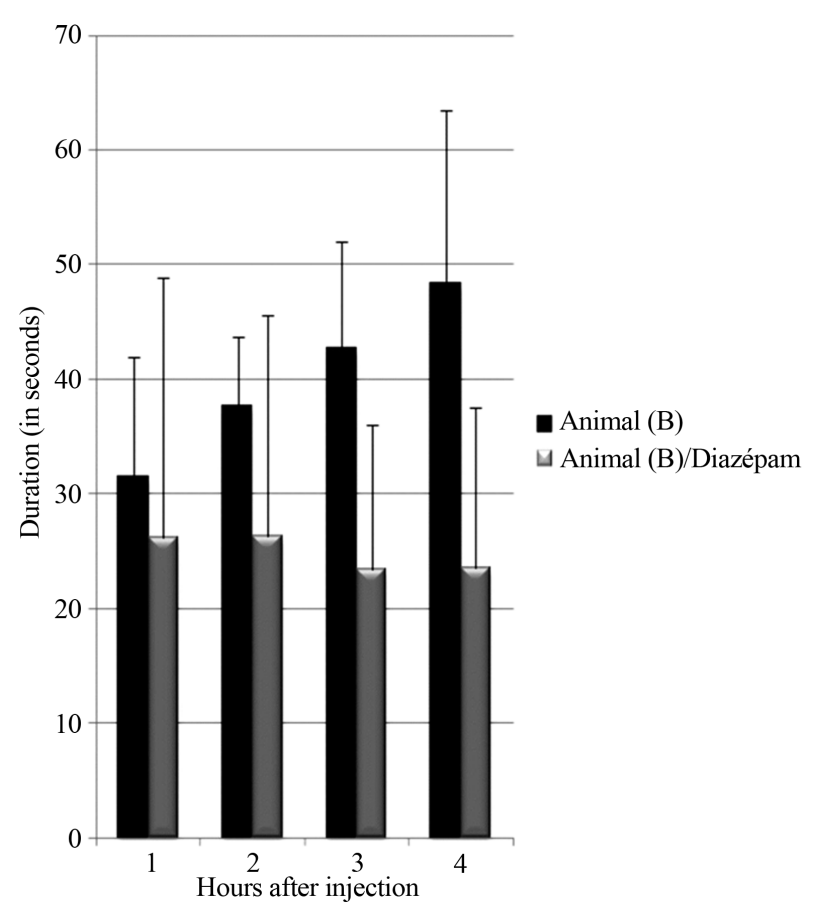

(b)

Figure 8. Effect of Diazepam on seizures Monkey B. Part A of figure shows effect of Diazepam on total duration of Seizures; Part B of figure shows effect of Diazepam on average duration of seizure in each hour. X-axis: Hours after intracortical injection Y-axis: Total time spent in seizures in seconds; Control experiments (Black bars) Diazepam (Grey bars). (a) Effect of diazepam on total time spent in seizure; (b) Effect of diazepam on average duration of seizure. 
(Figure 8(b)). The average number of seizures in each hour did decrease after administration of Diazepam.

The number of interictal spikes (myoclonic jerks) remained same even after the administration of Diazepam The frequency of myoclonus remained in the range of 60 to 210 in each hour in three experiments (Figure 9). It did not decrease significantly after administration of Diazepam.

\section{DISCUSSION}

In this study, we describe electrophysiological and clinical properties of a primate model of acute focal motor status epilepticus following intracortical injection of penicillin, which acts as a GABA receptor blocker. The main results of our study are: 1 ) we could obtain on demand, a stable and reproducible motor status; 2) the electro-clinical features of the seizures mimicked those seen in Kojelnikow syndrome; 3) the seizures were resistant to benzodiazepine although their number was significantly decreased. 4) no major histopathological lesions were seen on brain specimen.

Models of focal neocortical epilepsy secondary to topically applied penicillin have previously been described in large animals like cats [10], rabit [27], sheep [28] and monkey [29]. But there is a paucity of studies aimed at characterizing and quantifying semi-acute focal motor status epilepticus in primates. The development of such a model in primate is important because seizure progression might differ from species to species [30]. Critically, a primate model might be the most suited for preclinical testing of innovative therapies such as deep brain stimulation, local drug delivery, cooling of the epilepogenic zone or gene therapy, This was an important motivation in initiating this work. In fact subsequent to development of this model we have been successful in using it for testing Deep Brain Stimulation of basal ganglia in focal motor seizures We could maintain the modeled primates for several months and perform number of

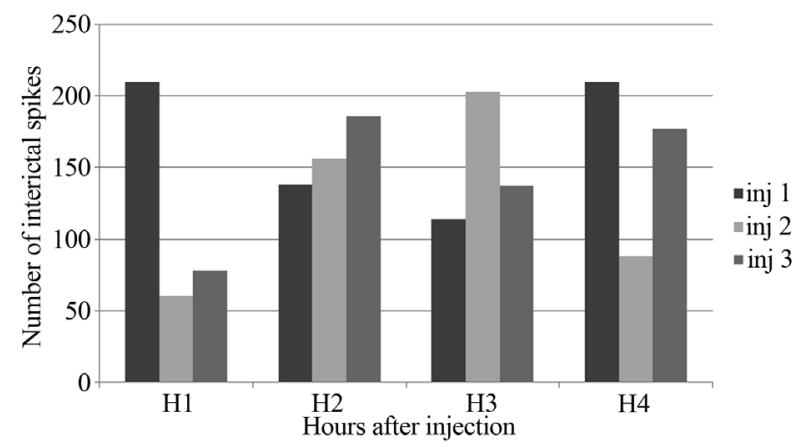

Figure 9. Number of interictal spikes during experiments with Diazepam. X-axis: Hours after intracortical injection; Y-axis: Number of interictal spikes. Inj: intracortical injection of Penicillin. experiments without deterioration of health of the animals or change in the characteristics of the model (data not shown).

\subsection{Clinical Semiology and Resistance to AED}

We found that focal intracortical injection of penicillin systematically led to a series of stereotypical and reproducible contralateral clinical signs that were strongly correlated with the ECoG spiking. These seizures closely mimicked those encountered in humans suffering from epilepsia partialis continua (EPC) and from Kojevnikov's syndrome.

Indeed, epilepsia partialis continua is described as a spontaneous regular or irregular clonic muscle twitching of cerebral cortical origin, confined to one part of the body and continuing for a period of hours, days, or even weeks [31]. In most of the cases of EPC, the seizures are of focal cortical origin and motor activity remains localized. Motor activity (myoclonus) is often persistent, lasting for at least 60 minutes and sometimes extending for hours, days even weeks. Consciousness usually is preserved but postictal weakness is frequently evident [32]. In Kojevnikov's syndrome, besides interictal spikes correlated with contralateral jerks, contralateral tonic seizures can occur. The clinical course seems to be not modified by use of anti epileptic drugs [33]. Failure of GABAergic inhibition and potentiation of excitatory (NMDA) synapses are considered as mechanisms for self-sustaining status epilepticus. This is also the reason for resistant to all AED (including benzodiazepine) except NMDA antagonists in such conditions [34].

The intracortical injection of penicillin pointing to a cortical origin of seizures, strictly regional clinical manifestation in contralateral limbs, relatively stable number of myoclonic jerks observed in the interictal period, contralateral and regional tonic seizures, resistance to Diazepam are features of our model which closely resemble the description of epilepsia partialis continua and Kojevnikov's syndrome.

However, there is a need to test response to other classes of AED and document focal changes at the injection site by histopathology in order to characterize this model as a representative of human motor epilepsy. Our model has the distinct advantage of being in the nonhuman primate thus incorporating anatomical pathways that may distinguish the primate (e.g. human) from rodent pathways and models. The semiology of the seizure bears strong resemblance to the human even if it does not replicate the pathology precisely nor the spontaneous aspects of human seizures. Furthermore, there is no underlying gross lesion (dysplasia, tumor, ischemic lesion) in the present model. However, our model is not a model of epilepsy syndrome but rather mimicks symptoms observed during motor seizures. 


\subsection{Comparison with Other Models}

In a sheep model of focal epilepsy induced by intra cortical injection of PNC, Opdam and colleagues [3] found that upto one third of the animals exhibited electroencephalographic seizures without any behavioral correlation.

This lack of motor response in association with the rhythmic ictal spiking may be due to methodological differences as Opdam et al. injected the penicillin in the premotor cortex, in contrast to direct motor cortex injection in our protocol.

Penicillin injection models in newborn monkeys have been described in the past [29]. However our model was characterized in adult monkeys and is suitable for pre clinical testing of therapies. Our model also differs from the more classical primate model of neocortical focal seizure obtained by intracortical application of alumina gel studied previously [30]. Indeed, in this model, the appearance of symptomatic epilepsy takes 4 - 8 weeks and behavioral characteristics are unpredictable with seizure frequency ranging from 1 per month to 10 per day (personal observation, unpublished data). Recently, focal seizures induced by electric cortical stimulation in primates has been studied to define optimal stimulation parameters for electroconvulsive therapy [35]. Though the seizures induced were focal as recorded on EEG, it was not possible to observe clinical response in this model because anesthetics and muscle relaxants were used. Interestingly, our model of focal motor seizure in monkeys share electrical and clinical resemblances with those observed in rats [4]. This might suggest that the protocol used in the present study can be replicated in others species.

\subsection{Reproducibility}

In our study, there was no secondary generalization of the seizures. The electrophysiological and behavioral abnormalities were usually absent after 24 hours after the injection. These are very attractive features of the model as they allow a rapid recovery of animal to a non epileptic state and improves safety in repeated experiments.

The various ECoG ictal patterns that were identified were reproducible even after several penicillin injections. The electrophysiological characteristics of this model are useful in evaluating treatment outcomes. Besides the usual duration and frequency of seizure, the number of interictal spikes, and polyspikes are tangible abnormalities that can be assessed as parameters for testing effectiveness. This is possible because the inter ictal spikes are observed both as an electric and a clinical event in the form of contraction of contralateral limb.

The time frequency analysis representative of the 17 seizures, indicated predominant beta rhythm activity throughout the course of a seizure. This beta rhythm, a marker of motor cortex activity, confirmed indirectly the site of intracortical injection of Penicillin

\subsection{Conclusions}

The PNC injection in motor cortex in primate leads to the induction of a reproducible acute focal motor status epilepticus. An animal model of epilepsy is considered suitable when it satisfies certain requisites. The model should be safe for the animal, simple to generate, easy to document and reproducible. Data obtained from ECoG recordings should be easy to use in automated analysis like automatic spike detection. Also for translational therapeutics of human diseases, the most important step is to validate novel therapies in closer species like primates.

Our model described above satisfies these conditions.

\section{ACKNOWLEDGEMENTS}

We wish to acknowledge the help provided by Sandrine Michallat during conduction of experiments.

\section{DECLARATION OF INTEREST}

All authors declare no conflict of interests related to this study. This work was supported by “Fondation de l'avenir” and Medtronic Europe. However these agencies had no role in designing and conduct of experiments.

\section{REFERENCES}

[1] Jobst, B. (2010) Brain stimulation for surgical epilepsy. Epilepsy Research 8, 154-161. http://dx.doi.org/10.1016/j.eplepsyres.2009.08.017

[2] Rogawski, M.A. (2009) Convection-enhanced delivery in the treatment of epilepsy: Nontraditional epilepsy treatment approaches. Neurotherapeutics, 6, 344-351. http://dx.doi.org/10.1016/j.nurt.2009.01.017

[3] Rothman, S.M., Matthew, D., Smyth, M.D., Yang, X.-F. and Peterson, G.P. (2005) Focal cooling for epilepsy: An alternative therapy that might actually work. Epilepsy \& Behavior, 7, 214-221. http://dx.doi.org/10.1016/j.yebeh.2005.05.021

[4] Chabardes, S., Najm, L. and Luders, H. (2008) Animal models of epilepsy relevant for epilepsy surgery studies. In: Luders, H. Ed., Handbook of Epilepsy Surgery, Infroma Healthcare, London, 1405-1413

[5] Pallud, J., Devergnas, A., Chabardes, S. and Depaulis, A. (2008) Animal models to develop surgery of focal epilepsies? Neurochirurgie, 54,128-134. http://dx.doi.org/10.1016/j.neuchi.2008.02.015

[6] Chevassus-au-Louis, N., Baraban, S.C., Gaiarsa, J.L. and Ben-Ari, Y. (1999) Cortical malformations and epi-lepsy: New insights from animal models. Epilepsia, 40, 811-821. http://dx.doi.org/10.1111/j.1528-1157.1999.tb00786.x

[7] De Deyn, P.P. and D’Hooge, R. (1999) Animal models of focal epilepsy. Acta Neurologica Belgica, 99, 222-225. 
[8] Fisher, R.S. (1989) Animal models of the epilepsies. Brain Research-Brain Research Reviews 14, 245-278. http://dx.doi.org/10.1016/0165-0173(89)90003-9

[9] Hashizume, K. and Tanaka, T. (1998) Multiple subpial transection in kainic acid-induced focal cortical seizure. Epilepsy Research, 32, 389-399. http://dx.doi.org/10.1016/S0920-1211(98)00065-5

[10] Yamamoto, K., Tanaka, T. and Yonemasu, Y. (1995) Jacksonian seizure model induced by a kainic acid mi-croinjection into unilateral sensori-motor cortex. Brain \& Nerve, 47, 477-483.

[11] Yang, X.F., Duffy, D.W., Morley, R.E. and Roth-man, S.M. (2002) Neocortical seizure termination by focal cooling: Temperature dependence and automated seizure detection. Epilepsia, 43, 240-245. http://dx.doi.org/10.1046/j.1528-1157.2002.33301.x

[12] Willmore, L.J., Sypert, G.W., Munson, J.V. and Hurd, R.W. (1978) Chronic focal epileptiform discharges induced by injection of iron into rat and cat cortex. Science 200, 1501-1503. http://dx.doi.org/10.1126/science.96527

[13] Eder, H.G., Jones, D.B. and Fisher, R.S. (1997) Local perfusion of diazepam attenuates interictal and ictal events in the bicuculline model of epilepsy in rats. Epilepsia, 38, 516-521. http://dx.doi.org/10.1111/j.1528-1157.1997.tb01134.x

[14] Stein, A.G., Eder, H.G., Blum, D.E., Drachev, A. and Fisher, R.S. (2000) An automated drug delivery system for focal epilepsy. Epilepsy Research, 39, 103-114. http://dx.doi.org/10.1016/S0920-1211(99)00107-2

[15] Johnson, H., et al. (1946) Effects of antibiotic sub-stances on the central nervous system. Archives of Neurology \& Psychiatry, 56, 184-197.

[16] Elger, C.E. and Speckmann, E.J. (1983) Penicil-lin-induced epileptic foci in the motor cortex: Vertical inhibition. Electroencephalography \& Clinical Neuro-physiology, 56, 604-622. http://dx.doi.org/10.1016/0013-4694(83)90028-7

[17] Gloor, P. (1969) Epileptogenic action of penicillin. Annals of the New York Academy of Sciences, 166, 350-360. http://dx.doi.org/10.1111/j.1749-6632.1969.tb46405.x

[18] Gloor, P., Quesney, L.F. and Zumstein, H. (1977) Pathophysiology of generalized penicillin epilepsy in the cat: the role of cortical and subcortical structures. II. Topical application of penicillin to the cerebral cortex and to subcortical structures. Electroencephalography \& Clinical Neurophysiology, 43, 79-94. http://dx.doi.org/10.1016/0013-4694(77)90198-5

[19] Matsumato, H. and Ajmonemarsan, C. (1964) Cellular mechanism in experimental epileptic seizure. Science, 144, 193-194. http://dx.doi.org/10.1126/science.144.3615.193

[20] Meyer, H. and Prince, D. (1973) Convulsant actions of penicillin: Effects on inhibitory mechanisms. Brain Research, 53, 477-482. http://dx.doi.org/10.1016/0006-8993(73)90237-0

[21] Noebels, J.L. and Prince, D.A. (1977) Presynaptic origin of penicillin after discharges at mammalian nerve terminals. Brain Research, 138, 59-74. http://dx.doi.org/10.1016/0006-8993(77)90784-3

[22] Anschel, D.J., Ortega, E. and Fischer, R.S. (2004) Diazepam prophylaxis for bicuculline-induced seizures: A rat dose-response model. Neurosciences Letters, 356, 6668. http://dx.doi.org/10.1016/j.neulet.2003.10.082

[23] Campbell, A.M. and Holmes, O. (1984) Bicuculline epileptogenesis in the rat. Brain Research, 323, 239-246. http://dx.doi.org/10.1016/0006-8993(84)90294-4

[24] Ajmone-Marsan, C. (1972) Focal electrical stimula-tion. In: Purpura, D.P., Penry, J.K., Tower D.B. and Woodburry, D. Eds., Experimental Models of Epilepsy-A Manual for the Laboratory Worker, Raven Press, New York, 147-172.

[25] Hiroshi, S., Najm, I., Boongird, A., Nair, D., Burrier, C. and Luders, H.O. (2002) High-frequency direct cortical stimulation and its production of after discharges. Epilepsia, 43.

[26] Le Van Quyen, M., lachaux, J., Rodriguez, E., Lutz, A., et al. (2001) Journal of Neurosciences Methods, 111, 8398. http://dx.doi.org/10.1016/S0165-0270(01)00372-7

[27] Pockberger H, Rappelsberger, P. and Petsche, H. (1984) Penicillin-induced epileptic phenomena in the rabbit's neocortex I. The development of interictal spikes after epicortical application of penicillin. Brain Research, 309, 241-246.

http://dx.doi.org/10.1016/0006-8993(84)90590-0

[28] Opdam, H.I., Federico, P., Jackson, G.D., Bu-chanan, J., Abbott, D.F., Fabinyi, G.C., Syngeniotis, A., Vosmansky, M., Archer, J.S., Wellard, R.M. and Bel-lomo, R. (2002) A sheep model for the study of focal epilepsy with concurrent intracranial EEG and functional MRI. Epilepsia, 43, 779-787. http://dx.doi.org/10.1046/j.1528-1157.2002.04202.x

[29] Kato, M., Malamut, B.L., Caveness, W.F., et al. (1980) Local cerebral glucose utilization in newborn and pubescent monkeys during focal motor seizures. Annals of Neurology, 7, 204-212. http://dx.doi.org/10.1002/ana.410070303

[30] Gale, K. (2004) The nigral control of epilepsy: Basal ganglia circuitry as a substrate for seizure control In: Luders, H.O., Ed., Deep Brain Stimulation and Epilepsy 1st Ed Martin Duniz, London and New York, pp. 87-96.

[31] Obeso, J.A., Rothwell, J.C. and Marsden, C.D. (1985) The spectrum of cortical myoclonus. From focal reflex jerks to spontaneous motor epilepsy. Brain, 108 (Pt 1), 193-124. http://dx.doi.org/10.1093/brain/108.1.193

[32] Bencaud, J., Boris. A. and Trottier, S. (1982) Continuous partial epilepsy: Syndrome and diseases. Revue Neurologique (Paris), 138, 803-814.

[33] Shomer, D.L. (1993) Focal status epilepticus and epilepsia partialis continua in adults and children. Epilepsia, 34, S29-S36. http://dx.doi.org/10.1111/j.1528-1157.1993.tb05904.x

[34] Mazarati, A.M. and Wasterlain, C.G. (1997) Blockers of NMDA receptor restore paired-pulse inhibition in the rat dentate gyrus lesioned by perforant path stimulation. Neuroscience Letters, 234, 135-138. http://dx.doi.org/10.1016/S0304-3940(97)00693-9 
[35] Spellman, T., Peterchev, A. and Lisanby, S. (2009) Focal electrically administered seizure therapy: A novel form of ECT illustrates the roles of current directionality, polarity, and electrode configuration in seizure induction. Neuropsychopharmacologym, 34, 2002-2010.

http://dx.doi.org/10.1038/npp.2009.12 